\title{
FINITE INDEX SUBGROUPS OF THE MODULAR GROUP AND THEIR MODULAR FORMS
}

\author{
LING LONG
}

\begin{abstract}
Classically, congruence subgroups of the modular group, which can be described by congruence relations, play important roles in group theory and modular forms. In reality, the majority of finite index subgroups of the modular group are noncongruence. These groups as well as their modular forms are central players of this survey article. Differences between congruence and noncongruence subgroups and modular forms will be discussed. We will mainly focus on three interesting aspects of modular forms for noncongruence subgroups: the unbounded denominator property, modularity of the Galois representation arising from noncongruence cuspforms, and Atkin and Swinnerton-Dyer congruences.
\end{abstract}

\section{INTRODUCTION}

The special linear group $S L_{2}(\mathbb{Z})$ consists of all 2-by-2 integral matrices with determinant 1 . It is one of the most well-known and important discrete groups. A finite index subgroup of the modular group is said to be congruence if it contains the kernel of the natural modulo $N$ homomorphism from $S L_{2}(\mathbb{Z})$ to $S L_{2}(\mathbb{Z} / N \mathbb{Z})$ for some positive integer $N$; otherwise, it is called a noncongruence subgroup. The existence of noncongruence subgroups of $S L_{2}(\mathbb{Z})$ was confirmed by Fricke [Fri86] and Pick [Pic86]. More noncongruence subgroups were constructed by Reiner [Rei58, Newman [New65], Rankin [Ran67, etc. In contrast, any finite index subgroup of $S L_{n}(\mathbb{Z})$ with $n \geq 3$ is congruence BLS64. A famous theorem of Belyı̆ implies any compact complex smooth irreducible curve defined over $\overline{\mathbb{Q}}$, the algebraic closure of $\mathbb{Q}$, can be realized (in many ways) as a modular curve (defined in 2.3) for a finite index subgroup of $S L_{2}(\mathbb{Z})$ [Bel79]. Consequently, finite index subgroups arise naturally in many fields such as the theory of dessin d'enfant which includes Galois coverings of the projective line [Bir94, triangular groups [Jon79, JS94, as well as the theory of translation surfaces HL05. As a matter of fact, noncongruence subgroups of $S L_{2}(\mathbb{Z})$ predominate congruence subgroups (Section 2.3 below).

Modular forms are generalizations of elliptic functions (doubly periodic functions) and enjoy plenty of wonderful properties. Many important one

The author was supported in part by an NSF-AWM mentoring travel grant for women. She would like to thank the Pennsylvania State University for hosting her visit during May-June 2006. 
variable formal power series arising in combinatorics or physics, such as the partition function, turn out to be closely related to modular forms.

The theory of congruence (elliptic) modular forms is now well-developed due to the efforts of many mathematicians. It has very broad impacts including some important applications to information technology [Sar90, Li96]. In the early days, computational observations and conjectures were significant driving forces for the development of this theory. For example, Ramanujan observed coefficients of $q \prod_{n \geq 1}\left(1-q^{n}\right)^{24}=\sum \tau(n) q^{n}$ satisfy special three-term recursions for small primes $p$

$$
\tau(n p)-\tau(p) \tau(n)+p^{11} \tau(n / p)=0, \forall n \in \mathbb{N},
$$

where $\tau(n / p)=0$ if $p \nmid n$. In [Mor17] Mordell showed that these recursions hold for all primes $p$ by using essentially Hecke operators. In [Hec37] Hecke defined Hecke operators and proved that they satisfy $(2 d+1)$-term relations on each $d$-dimensional space of congruence modular forms. Shortly after, Petersson introduced the Petersson inner product via which he showed the Hecke operators can be diagonalized simultaneously [Pet39]. The readers are referred to [LLY05a, Section 2] for a summary of the theory of newforms (congruence modular form with particular properties) developed by Atkin and Lehner [AL70], Miyake [Miy71, and Li [Li75]. The current considerable interest in modular forms is largely due to the vital role they played in the celebrated proof of Fermat's Last Theorem (FLT) by Wiles Wi195. In his proof, Wiles used a result of Frey and Ribet which says the Taniyama-Shimura-Weil (TSW) conjecture implies FLT. The TSW conjecture asserts the $l$-adic Galois representations attached to an elliptic curve (a genus one complete curve) defined over $\mathbb{Q}$ are isomorphic to the $l$-adic Deligne representations attached to a certain newform. The final proof of TSW ([Wil95, TW95, BCDT] ) is a major breakthrough in the very influential Langlands program which is a system of conjectures connecting the $l$-adic cohomology theory of algebraic varieties with representation theory of cuspidal automorphic forms Gel84, Mur93. In the sequel, we say an $l$-adic Galois representation is "modular" when it arises from a congruence automorphic form.

Modular forms for noncongruence subgroups have already been considered by Fricke and Klein. However, there was no serious investigation of these functions till the late 1960's as it was commonly believed that the Hecke theory was missing. Indeed, Atkin conjectured that Hecke operators defined classically have trivial actions on noncongruence modular forms and his conjecture was proven in part by Serre [Tho89] and Scholl [Sch97] and in general by Berger [Ber94].

However, the investigation in the past 40 years reveals the mathematical structure of noncongruence modular forms is still wonderfully rich. In [ASD71, Atkin and Swinnerton-Dyer used computers together with algebra and combinatorics to work with noncongruence subgroups and noncongruence modular forms. They brought up many interesting and fundamental ideas and 
original observations. In particular, they studied the arithmetic of the Fourier expansions $\sum a(n) w^{n}$ of noncongruence modular forms and observed that for some forms with algebraic coefficients the sequences $\{a(n)\}$ have unbounded denominators. On the other hand, it is known that if $f$ is a congruence holomorphic modular form, the sequence $\{a(n)\}$ must admit bounded denominators Shi71. This observation gives a simple and clear criterion for distinguishing noncongruence modular forms. Atkin and Swinnerton also found $p$-adic analogue of the Hecke three-term recursion by demonstrating the coefficients $\{a(n)\}$ of some noncongruence modular forms satisfy

$$
a(n p)-A(p) a(n)+B(p) a(n / p) \equiv 0, \forall n \geq 1
$$

modulo a power of $p$ depending on $\operatorname{ord}_{p}(n)$ for some constants $A(p)$ and $B(p)$. In ASD71 a proof of the weight 2 dimension 1 case was provided. The modularity of this case would have followed from the Taniyama-ShimuraWeil conjecture. For a few examples of dimension 2 or more, Atkin and Swinnerton-Dyer found that such three-term congruences still exist with the forms diagonalized $p$-adically for each separate $p$ and the $A(p)$ being over algebraic number fields in general different for each $p$. Several people ([Dit76, Sti85, etc]) studied Atkin and Swinnerton-Dyer (ASD) congruences. The general situation for the weight 2 cases has been confirmed by several papers [Hon70, Car71, Dit76, Kat81].

In [Sch85], using deep algebraic geometry and representation theory, Scholl associated each $d$-dimensional space of noncongruence cuspforms with $2 d$ dimensional $l$-adic Scholl representations and showed the existence of $(2 d+1)$ term $p$-adic recursions under general conditions. When $d=1$ Scholl fully established the 3-term ASD congruences (2). However when $d>1$, the existence of a fixed basis for each of which satisfies 3-term ASD congruences for all $p$ (referred to as simultaneous diagonalization) is rare.

Around the same time interesting connections between modular forms for congruence and noncongruence subgroups began to merge. In [Sch88, Sch93] Scholl gave examples of 1 dimensional spaces of cuspform with the $A(p)$ being eigenvalues of congruence cuspforms by showing the $l$-adic Scholl representations associated to those cases are "modular". In [Ber00, Berger found a quadratic relation satisfied by 2 noncongruence cuspforms and 2 congruence cuspforms.

In ASD71, Sch88 noncongruence subgroups with small indices are given considerable attention. Recently, investigation has been focused on the so called noncongruence character groups, which are "almost" congruence. These noncongruence subgroups are constructed by assigning characters to the generators of congruence subgroups. The Lattice groups considered by Rankin [Ran67] are examples of them. Klein and Fricke used "Wurzelmodul" to describe a Hauptmodul of a genus zero noncongruence character group defined 
by a cyclic character. Here, we will associate the Fermat curve $x^{n}+y^{n}=1$ with the modular curve for the intersection of two particular character groups.

In [LLY05b], Li et al. gave a 2-dimensional space of cuspforms for a noncongruence character group which satisfies simultaneous diagonalization. Moreover, it was shown that $A(p)$ are eigenvalues of explicit newforms. Later, Fang et al. found a few more examples of this kind [ $\left.\mathrm{F}^{+} 05\right]$. As a matter of fact, simultaneous diagonalization can rarely be achieved. More recently, Atkin et al [ALL05] and Long [Lon06] fully exhibited two cases which resemble the case in [LLY05b] but are slightly more general. In each case, a basis depending on the congruence class of $p$ modulo a fixed integer is found, for each of the basis function the coefficients satisfy a 3-term ASD congruence relation. Meanwhile, modularity of each case was verified individually. These modularity results rely heavily on the decompositions of the associated $l$-adic Scholl representations or their restrictions to suitable Galois subgroups. In contrast, Scholl exhibited other cases where the $l$-adic Scholl representations are not reducible [Sch04].

This expository paper is organized in the following order. Some background topics are introduced in Section 2. Section 3 is devoted to some differences between congruence and noncongruence subgroups. Modular forms and some related topics will be addressed briefly in Section 4. In Section 5, we will show the Fourier coefficients of some noncongruence modular forms have unbounded denominators. In Section 6 we will address $l$-adic Scholl representations attached to noncongruence cuspforms and related modularity results briefly. Section 7 is about the three-term Atkin-Swinnerton-Dyer congruence relations. Throughout this paper, several examples will be discussed and all proofs presented are elementary. The author has not made an attempt to give a comprehensive review of the vast literature. This survey mostly reflects the author's perspectives on these topics based on her recent research activities.

This paper has grown out of the author's talk delivered at the Banff conference. The author is indebted to A.O.L. Atkin. The introduction of this article is written partly based on the valuable historic recounting and analysis of the development of noncongruence modular forms by A.O.L. Atkin (communicated through private correspondence). His wisdom and original ideas have sparked much of the discussion here. It is the author's great pleasure to thank Wenching Winnie Li for her constant discussions and many insightful comments. Some results below are cited from some unpublished notes of Li and the author. The author would also like to thank the conference organizers and in particular Noriko Yui for inviting her to attend this wonderful conference and giving her an opportunity to present this talk. She is also very grateful to many people such as Ron Livné, Siu-Hung Ng, Jan Stienstra, Yifan Yang, Don Zagier, for their valuable discussions and comments. The author specially thanks Chris Kurth for his remarks on an earlier version of 
this paper, Tonghai Yang for pointing out several useful references, and the referee for many suggestions on improving the clarity of this paper.

\section{BACKGROUNDS}

In this article, we use $p$ and $l$ for prime numbers and $\mathbb{Q}_{l}$ for the field of $l$-adic numbers. We adapt mostly common notations. We use $w$ for all local parameter at infinity instead of $q^{\mu}$ where $\mu$ varies in different cases.

2.1. Finite index subgroups of $P S L_{2}(\mathbb{Z})$. It is well-known that $S L_{2}(\mathbb{Z})$ is generated by two elements $E=\left(\begin{array}{cc}0 & 1 \\ -1 & 0\end{array}\right)$ and $V=\left(\begin{array}{cc}1 & 1 \\ -1 & 0\end{array}\right)$ [Ran77]. Identifying elements in $S L_{2}(\mathbb{Z})$ which differ by a sign, one obtains the modular group $P S L_{2}(\mathbb{Z})$. It is well-known that $P S L_{2}(\mathbb{Z})$ is the free-product of two elements with orders 2 and 3 respectively, namely the images of $E$ and $V$ in $P S L_{2}(\mathbb{Z})$ Ran77. In this article, we use $\Gamma$ to denote finite index subgroups of $S L_{2}(\mathbb{Z})$. We also use $\Gamma$ to denote the image of $\pm \Gamma / \pm I$ in $P S L_{2}(\mathbb{Z})$ if there is no confusion.

Congruence subgroups are a class of subgroups which are easy to describe. For example, given a positive integer $n$, the following are some well-known congruence subgroups.

$$
\begin{aligned}
& \Gamma^{0}(n)=\left\{\gamma \in S L_{2}(\mathbb{Z}), \gamma=\left(\begin{array}{ll}
* & 0 \\
* & *
\end{array}\right) \quad \bmod n\right\} . \\
& \Gamma^{1}(n)=\left\{\gamma \in S L_{2}(\mathbb{Z}), \gamma=\left(\begin{array}{ll}
1 & 0 \\
* & 1
\end{array}\right) \quad \bmod n\right\} \text {. } \\
& \Gamma(n)=\left\{\gamma \in S L_{2}(\mathbb{Z}), \gamma=\left(\begin{array}{ll}
1 & 0 \\
0 & 1
\end{array}\right) \quad \bmod n\right\} .
\end{aligned}
$$

In Mil69a, Mil69b, Millington showed that up to isomorphism there is a one-to-one correspondence between finite index subgroups of $P S L_{2}(\mathbb{Z})$ and a pair of permutations of orders 2 and 3 respectively which generate a finite transitive permutation group.

Example 1. The group $\Gamma^{1}(3)$ is an index 4 subgroup of $S L_{2}(\mathbb{Z})$. It has 4 right coset representatives $\gamma_{1}=\left(\begin{array}{ll}1 & 1 \\ 0 & 1\end{array}\right), \gamma_{2}=\left(\begin{array}{ll}1 & 2 \\ 0 & 1\end{array}\right), \gamma_{3}=\left(\begin{array}{ll}1 & 3 \\ 0 & 1\end{array}\right), \gamma_{4}=$ $\left(\begin{array}{cc}0 & -1 \\ 1 & 0\end{array}\right)$. For simplicity, we will use $1,2,3,4$ to denote the corresponding cosets. Multiplying $E$ on the right will permute the right cosets. More explicitly, $E=(12)(34)$ is an order 2 permutation. Similarly, $V$ corresponds to an order 3 permutation $V=(134)(2)$. Millington's theorem says $\Gamma^{1}(3)$ corresponds uniquely (up to isomorphism) to the pair of permutations $(12)(34)$ and $(134)(2)$. Note that in this case $\left(\begin{array}{cc}1 & -1 \\ 0 & 1\end{array}\right)=V E=(123)(4)$. Since the 
group of stabilizers of the cusp $\infty$ is generated by $\left(\begin{array}{cc}1 & -1 \\ 0 & 1\end{array}\right)$, the corresponding cycle (123) is called the marked cycle in the disjoint cycle representation of $V E$ with respect to $\infty$.

2.2. KFarey. The permutation representation of these groups is of great importance to the computational aspects of finite index groups of the modular group. For example, in [Hsu96], Hsu gave an algorithm for identifying congruence subgroups based on the permutation representations. Recently, Kurth has developed a SAGE package called "KFarey" which has implemented many useful functions for finite index subgroups such as Hsu's algorithm mentioned above, Farey symbols by Kulkarni [Kul85], determining whether a given element is in a group specified by a given permutation representation and a "marked" cycle in the permutation representation of $V E$ corresponding to infinity, etc. The "KFarey" can be downloaded from the following website

\section{http://www.public.iastate.edu/kurthc/research/index.html}

2.3. Modular curves. The elements $\left(\begin{array}{ll}a & b \\ c & d\end{array}\right) \in \Gamma$ act on the upper half plane $\mathfrak{H}=\{z \in \mathbb{C} \mid \operatorname{Im} z>0\}$ via the linear fractional transformation:

$$
\left(\begin{array}{ll}
a & b \\
c & d
\end{array}\right) \cdot z=\frac{a z+b}{c z+d}, \quad \forall z \in \mathfrak{H} .
$$

An element $\gamma=\left(\begin{array}{ll}a & b \\ c & d\end{array}\right) \in S L_{2}(\mathbb{Z})$ is said to be elliptic (resp. parabolic or hyperbolic) if the value of $|a+d|$ is $<2$ (resp. $=2$ or $>2$ ). Each elliptic (resp. parabolic) element has a unique fixed point on $\mathfrak{H}$ (resp. $\mathbb{Q} \cup\{\infty\}$ ). Hyperbolic elements have no fixed points on $\overline{\mathfrak{H}}=\mathfrak{H} \cup \mathbb{Q} \cup\{i \infty\}$. (Whether a group has elliptic elements or not can be easily read off from its permutation representation. For example, $\Gamma^{1}(3)$ has order 3 elliptic points as the left coset labeled by 2 is fixed by $V$.) The orbit space $\mathfrak{H} / \Gamma$ can be compactified canonically by adding a few isolated points called the cusps of $\Gamma$ (equivalence classes in $(\{\infty\} \cup \mathbb{Q}) / \Gamma)$. The compactified curve, denoted by $X_{\Gamma}$ is called the modular curve associated with $\Gamma$. By convention, the genus of $X_{\Gamma}$ is also called the genus of $\Gamma$. For convenience, we will assume that all modular curves here are defined over $\mathbb{Q}$ unless specified otherwise. When the curve $X_{\Gamma}$ has genus 0 , the field of meromorphic functions on $X_{\Gamma}$ is generated by a single element, which is called a Hauptmodul of $\Gamma$. For example, the classical modular $j$-function is a Hauptmodul of $S L_{2}(\mathbb{Z})$.

The importance of finite index subgroups of $S L_{2}(\mathbb{Z})$ is seen in the following theorem.

Theorem 1 (Belyi [Bel79]). Any smooth compact complex projective curve defined over $\overline{\mathbb{Q}}$ is isomorphic to the modular curve of some finite index subgroup of $S L_{2}(\mathbb{Z})$. 
On the other hand, one should know that the above correspondence is not unique. Please refer to Section 5.5 for a connection between Fermat curves and modular curves which demonstrates Belyi's theorem above.

Up to conjugation, the stabilizer of each cusp $c$ of $\Gamma$ is generated by a matrix of the form $\pm\left(\begin{array}{cc}1 & m \\ 0 & 1\end{array}\right)$, where $m \geq 1$ is called the cusp width of $c$. The level of the group $\Gamma$, denoted by $\operatorname{lev}(\Gamma)$, is the least common multiple of all cusp widths of $\Gamma$. It was defined by Wohlfahrt [Woh64] which generalizes Klein's level definition for congruence subgroups.

\subsection{Elliptic modular surface. An elliptic surface}

$$
\pi: S \rightarrow C
$$

is a two dimensional complex variety $S$ together with a morphism map $\pi$ to the base curve $C$ such that for almost all $t \in C, \pi^{-1}(t)$ is an elliptic curve. When $\pi^{-1}(c)$ is not an elliptic curve, it is called a special fiber. Special fibers of the Néron models of elliptic surfaces have been classified by Kodaira [Kod63] and Néron [Nér64. An elliptic surface $\pi: S \rightarrow C$ is called semistable if all of its special fibers are of $I_{n}$ type (in Kodaira notation) and the list of the (un-ordered) numbers $\left[n_{1}, n_{2}, \cdots, n_{r}\right]$ in the subscripts of the special fibers is called the configuration of the semistable elliptic surface.

Let $\mathbb{C}$ be the ground field. Given an elliptic fibration $\pi: S \rightarrow C$, let $\Sigma$ be the finite collection of points on $C$ corresponding to special fibers. Via a standard procedure (cf. Kod63]) one obtains the monodromy representation of the fundamental group $\pi_{1}\left(C \backslash \Sigma, t_{0}\right)$ :

$$
\rho_{\pi}: \pi_{1}\left(C \backslash \Sigma, t_{0}\right) \rightarrow \mathrm{GL}_{2}(\mathbb{C}),
$$

where $t_{0}$ is a fixed reference point on $C \backslash \Sigma$. The image of $\rho_{\pi}$, well-defined up to a conjugation corresponding to the difference choices of $t_{0}$, is called the monodromy group of $\pi$. Nori gave criterions for when the monodromy group of an elliptic surface $\pi: S \rightarrow C$ gives rise to a finite index subgroup of the modular group [Nor85]. By Nori's theorem, the monodromy group of a semistable elliptic surface with maximal possible Picard number is a finite index subgroup of $S L_{2}(\mathbb{Z})$. Here for a surface $S$, the $\mathbb{Z}$-rank of its group of divisors modulo linear equivalence is called the Picard number of $S$.

In Shi72, for every finite index subgroup $\Gamma$ of $S L_{2}(\mathbb{Z})$ not containing $-I_{2}$, Shioda constructed an elliptic modular surface $\pi: \mathcal{E}_{\Gamma} \rightarrow X_{\Gamma}$ associated with $\Gamma$. In particular, for an index 24 genus zero subgroup $\Gamma, \mathcal{E}_{\Gamma}$ is an elliptic K3 surface. K3 surfaces are simply connected compact complex surfaces with trivial canonical bundles. They are two dimensional Calabi-Yau manifolds and occupy an important role in the classification of compact complex surfaces (cf. [BPVdV84]). Miranda and Persson have classified all configurations of semistable elliptic K3 surfaces with Picard number 20 [MP89]. Each K3 surface of this kind corresponds to an index 24 subgroup with 6 cusps and 
its cusp widths are given by the configuration of the surface. (In [MB], Montanus and Beukers computed algebraic models of those semistable elliptic K3 surfaces defined over $\mathbb{Q}$.) Among the 112 possible extremal semistable K3 configurations, 9 cases correspond to congruence subgroups. In [TY03], Topp and Yui have mentioned that among the remaining 103 configurations corresponding to noncongruence subgroups, 10 can be obtained via double covers of the semistable extremal rational elliptic surfaces classified by Beauville Bea82 ramifying at only two points. For example, a semistable K3 with a configuration $[1,1,1,1,10,10]$ is a double cover of a semistable rational surface with a configuration $[1,1,5,5]$ (cf. Example 2 below).

2.5. Most finite index subgroups are noncongruence. Rademacher conjectured that there are only finitely many genus zero congruence subgroups (c.f [KN65]). In [Den75], Dennin showed that there are only finitely many congruence subgroups of a given genus. In [Seb01], Sebber classified all elliptic elements free genus zero congruence subgroups. There are 33 such groups in total. In CLY04, Chua, Lang, and Yang gave explicit descriptions for all the genus zero congruence subgroups. In CP03, Cummins and Pauli classified all congruence subgroups with genus less than 25 .

On the other hand, Newman showed that there are infinitely many genus zero noncongruence subgroups [New65]. In [ASD71, Atkin and SwinnertonDyer pointed out that noncongruence subgroups predominate congruence subgroups. In [Jon79, Jones proved that there are infinitely many noncongruence subgroups of a given genus (cf. also [Jon86]). Let $N_{c}(n)$ (resp. $N_{n c}(n)$ ) be the number of congruence (resp. noncongruence) subgroups of index $n$ in $P S L_{2}(\mathbb{Z})$. Stothers gave an upper bound for $N_{c}(n)$ and showed that $N_{c}(n) / N_{n c}(n) \rightarrow 0$ when $n \rightarrow \infty$ [Sto84].

2.6. Noncongruence character groups. It is well-known that for any positive integer $n>2$, the finite index subgroup $\Gamma^{1}(n)$ of $S L_{2}(\mathbb{Z})$ is a normal subgroup of $\Gamma^{0}(n)$ with the quotient group $\Gamma^{0}(n) / \Gamma^{1}(n)$ isomorphic to $(\mathbb{Z} / n \mathbb{Z})^{\times}$. Such a relation provides a canonical decomposition for the spaces of modular forms (cf. Section 4 ) for $\Gamma^{1}(n)$ in terms of the characters of $(\mathbb{Z} / n \mathbb{Z})^{\times}$(cf. [Kob93]). In the same spirit, character groups are defined as follows:

Definition 1. A group $\Gamma$ is called a character group of a finite index subgroup $\Gamma^{0}$ if $\Gamma$ is normal in $\Gamma^{0}$ with abelian quotient. Equivalently, a character group is the kernel of an onto homomorphism

$$
\varphi: \Gamma^{0} \rightarrow G
$$

where $G$ is a finite abelian group written multiplicatively.

By the Fundamental Theorem of Finite Abelian Groups, it suffices to consider when $\Gamma^{0} / \Gamma$ is cyclic.

When working with character groups, we distinguish the following two types of character groups. 
Definition 2. A character group $\Gamma=\operatorname{ker} \varphi$ is said to be of type I if $\varphi(\gamma) \neq 1$ for some parabolic $\gamma \in \Gamma^{0}$; otherwise, it is said to be of type II. $\Gamma$ is said to be of type $\mathrm{II}(\mathrm{A})$, if the images of all elliptic and parabolic elements of $\Gamma^{0}$ are 1 under $\varphi$.

Note that if $\Gamma$ is a type II character group of $\Gamma^{0}$ then the levels of $\Gamma$ and $\Gamma^{0}$ are the same; this is not true for type I character groups in general. It has been shown that almost all type II character groups are noncongruence [KL06. If $\Gamma$ is a type $\mathrm{II}(\mathrm{A})$ character group of $\Gamma^{0}$, then the modular curve for $\Gamma$ is a strictly $\left[\Gamma^{0}: \Gamma\right]$ to 1 unramified covering map of the modular curve for $\Gamma^{0}$. By definition, the image of any elliptic element $\gamma$ in $\Gamma^{0}$ under $\varphi$ has to divide the order of $\gamma$ which is a finite positive integer.

In [LLY05b, ALL05, Lon06], the following family of type I character groups is considered.

Example 2. Let

$$
\Gamma^{1}(5)=\left\{\gamma \in S L_{2}(\mathbb{Z}) \mid \gamma \equiv\left(\begin{array}{ll}
1 & 0 \\
* & 1
\end{array}\right) \quad \bmod 5\right\} .
$$

This is an index 12 elliptic element free congruence subgroup of $S L_{2}(\mathbb{Z})$ with 4 cusps: $0,-5 / 2,-2, \infty$. The surface $\mathcal{E}_{\Gamma^{1}(5)}$ is semistable with configuration $[1,1,5,5]$. It is one of the 6 semistable extremal rational surfaces classified by Beauville Bea82. This group is generated by 4 elements denoted by $\gamma_{\infty}, \gamma_{-2}, \gamma_{0}, \gamma_{-5 / 2}$ (each stabilizes one cusp as indicated by the subscripts) subject to one relation

$$
\gamma_{\infty} \gamma_{-2} \gamma_{0} \gamma_{-5 / 2}=I_{2}
$$

Let $\varphi_{n}$ be the homomorphism from $\Gamma^{1}(5)$ to $\mathbb{C}^{\times}$sending $\gamma_{\infty}$ to $\omega_{n}, \gamma_{-2}$ to $\omega_{n}^{-1}$, and $\gamma_{0}, \gamma_{-5 / 2}$ both to 1 , where $\omega_{n}=e^{2 \pi i / n}$. The kernel of $\varphi_{n}$, denoted by $\Gamma_{n}$, is a type I character group of $\Gamma^{1}(5)$. We list a few properties of $\Gamma_{n}$ here:

1. $\Gamma_{n}$ is noncongruence when $n \neq 1,5$ (cf. Section 5).

2. $\Gamma_{n}$ is elliptic element free with index $12 n$ in $S L_{2}(\mathbb{Z})$ and has $2+2 n$ cusps.

3. $\Gamma_{5}$ is the last group in Sebbar's list Seb01.

4. The elliptic modular surface for $\Gamma_{2}$ is a $K 3$ surface with configuration $[1,1,1,1,10,10]$.

Example 3. The group $\Gamma^{0}(11)$ is genus 1, torsion free, and index 12 in $S L_{2}(\mathbb{Z})$. It has two cusps: $\infty$ and 0 . It is generated by 4 generators: $g_{\infty}$ and $g_{0}$ which stabilize $\infty$ and 0 respectively and two parabolic generators $A$ and $B$ subject to one condition

$$
g_{\infty} g_{0} A B A^{-1} B^{-1}=I_{2} .
$$

Then the kernel of any homomorphism which sends $g_{\infty}$ and $g_{0}$ to the identity and $A$ and $B$ to roots of unity is a type II character of $\Gamma^{0}(11)$. Among all such groups, there is only one, namely $\Gamma^{1}(11)$, which is congruence. By 


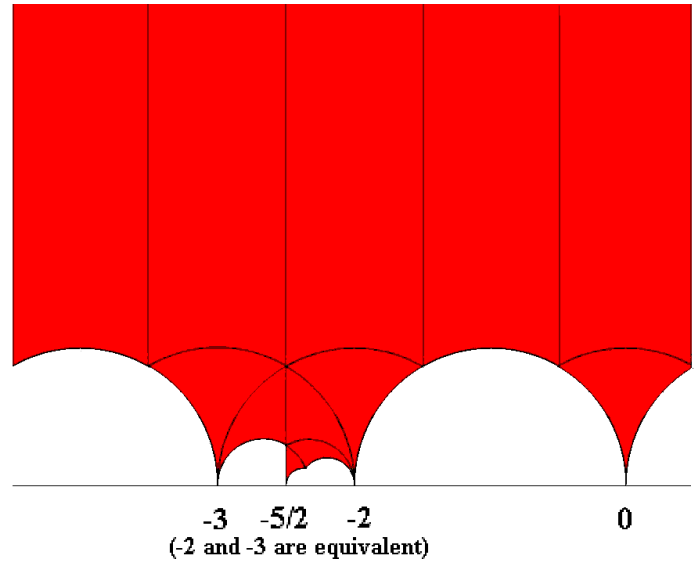

Figure 1. Fundamental domain for $\Gamma^{1}(5)$, drawn by the Fundamental Domain Drawer of Verrill

the Hurwitz formula, the genus of such a group remains 1 and as we have remarked before the level of this group is 11 . For any type II character group $\Gamma$ of $\Gamma^{0}(11)$, its modular curve $X_{\Gamma}$ is an elliptic curve which is isogenous to $X_{\Gamma^{0}(11)}$.

\section{Some Distinctions BetweEn CONGRUEnCE AND NONCONGRUENCE SUBGROUPS}

3.1. One fundamental difference between congruence and noncongruence subgroups is that elements of the congruence subgroups can be described by congruence relations while the elements in the noncongruence subgroups cannot.

3.2. Conjecturally, Fourier coefficients of modular forms for these two types of groups also behave differently. Please refer to Section 5 for a more detailed discussion.

3.3. The following is a special case of the finiteness theorem of Thompson [Tho80]. Let $\Gamma$ be a finite index subgroup of the modular group and $\mathcal{K}$ the set of congruence subgroups of $\Gamma$, then

$$
\lim _{K \in \mathcal{K}} \frac{g(K)}{|\Gamma: K|}=\frac{1}{2} \chi(\Gamma)
$$

in the sense that for any $\varepsilon>0$, there are only finitely many elements $K \in \mathcal{K}$ such that

$$
\left|\frac{g(K)}{|\Gamma: K|}-\frac{1}{2} \chi(\Gamma)\right|>\varepsilon .
$$


Here $g(\Gamma)$ is the genus of $\Gamma$,

$$
\chi(\Gamma)=2(g(\Gamma)-1)+t+\sum_{i=1}^{m}\left(1-\frac{1}{e_{i}}\right),
$$

is the Euler characteristic of $\Gamma, t$ is the number of cusps of $\Gamma, m$ is the number of elliptic points of $\Gamma$, and the $e_{i}$ 's are the orders of the elliptic points in $P S L_{2}(\mathbb{Z})$.

In comparison, there are infinitely many noncongruence subgroups of a given genus [Jon79]. Thus, the finiteness theorem of Thompson [Tho80] does not hold if the congruence condition on the set of elements in $\mathcal{K}$ is removed.

3.4. Let $A(\mathfrak{H} / \Gamma)$ denote the hyperbolic area of a fundamental domain of $\Gamma$. Let

$$
\Delta=-4(\operatorname{Im} z)^{2} \frac{\partial^{2}}{\partial z \partial \bar{z}}
$$

be the Laplace operator of the Poincaré metric on $\mathfrak{H}$. It admits a self-adjoint non-negative extension in the space of square integrable functions on $\mathfrak{H} / \Gamma$, which is denoted by $\Delta_{\Gamma}$. Let $\lambda_{1}$ be the smallest positive discrete eigenvalue of $\Delta_{\Gamma}$ (if it exists).

Theorem 2 (Selberg [Sel65]). If $\Gamma$ is a congruence subgroup, then $\lambda_{1} \geq 3 / 16$.

Theorem 3 (Zograf [Zog91]). Let $\Gamma$ be a discrete subgroup of $P S L_{2}(\mathbb{R})$ with hyperbolic area $A(\mathfrak{H} / \Gamma)<\infty$, and assume $A(\mathfrak{H} / \Gamma) \geq 32 \pi \cdot(g(\Gamma)+1)$. Then $\lambda_{1}$ exists and

$$
\lambda_{1}<\frac{8 \pi \cdot(g(\Gamma)+1)}{A(\mathfrak{H} / \Gamma)} .
$$

Theorem 4. For any $\varepsilon>0$, there exists a noncongruence subgroup with $0<\lambda_{1}<\varepsilon$.

Proof. As there are genus 0 noncongruence subgroups with $A(\mathfrak{H} / \Gamma)$ arbitrarily large (cf. Example 2), the claim follows from (6).

\section{Modular Forms}

4.1. A holomorphic (or meromorphic) modular form $f$ for $\Gamma$ is a holomorphic (or meromorphic) function defined on $\overline{\mathfrak{H}}$ satisfying

$$
f\left(\frac{a z+b}{c z+d}\right)=(c z+d)^{k} f(z), \quad \forall z \in \mathfrak{H},\left(\begin{array}{ll}
a & b \\
c & d
\end{array}\right) \in \Gamma .
$$

The number $k$ is called the weight of the modular form $f$. A weight 0 meromorphic modular form is called a modular function for $\Gamma$. All modular functions for $\Gamma$ together form a field, denoted by $\mathfrak{M}_{\Gamma}$, which is transcendental over $\mathbb{C}$. When $\Gamma$ has genus zero, $\mathfrak{M}_{\Gamma}$ is generated by any Hauptmodul for $\Gamma$.

A holomorphic modular form is called a cuspform if it vanishes at every cusp of $\Gamma$. Let $S_{k}(\Gamma)$ denote the space of holomorphic weight $k$ cuspforms 
for $\Gamma$. It is a finite dimensional vector space over $\mathbb{C}$. Explicit formulae for the dimension of $S_{k}(\Gamma)$ are available in [Shi71]. Cuspforms are important invariants of $\Gamma$ and $X_{\Gamma}$. For instance weight 2 cuspforms for $\Gamma$ are invariant differential 1-forms of $X_{\Gamma}$. A cuspform of integral weight larger than 2 is a differential form on $X_{\Gamma}$ with coefficients in a line bundle.

For any $\gamma=\left(\begin{array}{ll}a & b \\ c & d\end{array}\right) \in S L_{2}(\mathbb{Z})$, define the stroke operator $\left.\right|_{\gamma}$ action on a weight $k$ modular form $f$ to be

$$
\left.f\right|_{\gamma}=(c z+d)^{-k} f\left(\frac{a z+b}{c z+d}\right) .
$$

Theta functions and Eisenstein series constitute important classes of modular forms. They are described in most books on modular forms (Ogg69, Shi71, Kob93, DS05, etc]). In this paper, we use $w$ to denote $e^{2 \pi i z / \mu}$ where $\mu$ is the width of the cusp at infinity. Accordingly, any modular form has an expansion of the form $\sum_{n \geq n_{0}} a(n) w^{n}$.

Example 4. There are two particular holomorphic weight 3 Eisenstein series for $\Gamma^{1}(5): E_{1}$ and $E_{2}$, which vanish at all the cusps of $\Gamma^{1}(5)$ except $\infty$ and -2 respectively. Both $E_{1}$ and $E_{2}$ have integral Fourier expansions at $\infty$. Moreover, $E_{1} \cdot E_{2}=\eta(5 z)^{3} \cdot \eta(z)^{9}$, here $\eta(z)=q^{1 / 24} \prod_{n \geq 1}\left(1-q^{n}\right), q=e^{2 \pi i z}$ is the Dedekind eta function. One interesting expression for $E_{1}$ is

$$
E_{1}=1-\sum_{n \geq 1} b_{n} \frac{n^{2} w^{n}}{1-w^{n}}
$$

where $b_{n}=2,1,-1,-2,0,2,1,-1,-2,0 \cdots$ Sti05].

A Hauptmodul of $\Gamma^{1}(5)$ is

$$
t=\frac{E_{1}}{E_{2}}=w^{-1}+5+10 w+5 w^{2}-15 w^{3}-24 w^{4}+15 w^{5}+\cdots, \quad w=e^{2 \pi i z / 5} .
$$

Another formula for $t$ is (cf. [Seb02])

$$
t=\frac{1}{w} \prod_{n \geq 1}\left(1-w^{n}\right)^{-5\left(\frac{n}{5}\right)},
$$

where $(\dot{\overline{5}})$ is the Legendre symbol.

A Hauptmodul for $\Gamma_{n}$ is

$$
t_{n}=\sqrt[n]{t}
$$

In particular, it is also known that $t_{5}$ is a Hauptmodul for $\Gamma(5)$ (when $\left.w=e^{2 \pi i z}\right)$.

4.2. Cuspforms for noncongruence character groups. Due to the definition of noncongruence character groups, modular forms for such a group $\Gamma$ are closely related to modular forms for $\Gamma^{0}$. The following example illustrates such a relation. When $k>3$ we have the following result. 
Lemma 1. Let $\Gamma_{n}$ be as in Example 2. Let $k>3$ and $f_{n, k} \in S_{k}\left(\Gamma^{1}(5)\right)$ with simple zeros at the cusps $-2,0,-5 / 2$ and an order $k-3$ zero at infinity (and no other zeros). Then

$$
f_{n, k} \cdot t_{n}^{j}, j=1-n, \cdots,(2 k-3) n-1 .
$$

form a basis of $S_{k}\left(\Gamma_{n}\right)$.

Proof. Using the dimension formula in [Shi71], we have $\operatorname{dim} S_{k}\left(\Gamma_{n}\right)=2 n(k-$ $1)-1$. By the Riemann-Roch theorem, such a cuspform $f_{n, k}$ exists and for any $j \in\{1-n, \cdots,(2 k-3) n-1\}, f_{n, k} \cdot t_{n}^{j} \in S_{2 k}\left(\Gamma_{n}\right)$. As these functions are linearly independent, they form a basis.

4.3. Hecke operators. Many good properties of congruence modular forms are derived from Hecke operators. The major difficulty in understanding modular forms for noncongruence subgroups is the lack of satisfactory Hecke operators. For a finite index subgroup $\Gamma$, define the $p$ th Hecke operator $T_{p, \Gamma}$ associated to $\Gamma$ as follows: Let $\alpha_{p}=\left(\begin{array}{ll}p & 0 \\ 0 & 1\end{array}\right)$. We decompose the set $\Gamma \alpha_{p} \Gamma=\left\{\gamma \alpha_{p} \gamma^{\prime} \mid \gamma, \gamma^{\prime} \in \Gamma\right\}$ into a finite disjoint union $\bigcup_{i} \Gamma \alpha_{i}$. For any modular form $f$ for $\Gamma$, define

$$
T_{p, \Gamma} f=\left.\sum f\right|_{\alpha_{i}} .
$$

It is conjectured by Atkin and has been shown by Serre (for $\Gamma$ normal in $S L_{2}(\mathbb{Z})$ [Tho89]), Scholl (for certain cases [Sch97]), and Berger (in general Ber94]) that

Theorem 5. For any prime $p \nmid \operatorname{lev}(\Gamma)$ and all $f \in S_{k}(\Gamma)$

$$
T_{p, \Gamma} f=T_{p, \Gamma^{0}} \circ \operatorname{Tr}_{\Gamma}^{\Gamma^{0}} f,
$$

where $\operatorname{Tr}_{\Gamma}^{\Gamma^{0}}: S_{k}(\Gamma) \rightarrow S_{k}\left(\Gamma^{0}\right)$ is the trace map.

Consequently for all $f \in S_{k}(\Gamma) \backslash S_{k}\left(\Gamma^{0}\right), T_{p, \Gamma} f=0$.

\section{UNBOUNDED DENOMINATORS}

5.1. In what follows, we only consider modular forms $\sum_{n \geq n_{0}} a(n) w^{n}$ with coefficients $a(n)$ in a fixed number field. Due to Hecke operators, each space of holomorphic congruence modular forms has a basis consisting of forms with integral coefficients Shi71. Consequently, for every congruence holomorphic modular form with algebraic coefficients, the sequence $\{a(n)\}$ has bounded denominators in the sense that there exists an algebraic number $M$ such that $M \cdot a(n)$ is algebraic integral for all $n$. In [ASD71], Atkin and Swinnerton observed that this is no longer the case for noncongruence modular forms. Therefore, the sequence $\{a(n)\}$ having unbounded denominators clearly implies $f=\sum_{n \geq n_{0}} a(n) w^{n}$ is noncongruence. At present, it remains unknown whether $\{a(n)\}$ having bounded denominators will imply $\sum a(n) w^{n}$ to be congruence. We will formulate a more precise conjecture as follows: 
Let (UBD) refer to the following property:

Let $f$ be an arbitrary holomorphic integral weight modular form for $\Gamma$ but not for $\Gamma^{0}$ with algebraic Fourier coefficients at infinity. Then the Fourier coefficients of $f$ have unbounded denominators.

An open question is:

Does every noncongruence subgroup satisfy the condition (UBD)?

Remark 1. In [ASD71] and [Sch85], the coefficients $a(n)$ are normalized so that they are contained in smaller fields. In the following discussion, we have avoided the normalization on purpose for the following two reasons. First, such a procedure is unnecessary for all cases below. Second, it is more appropriate to address the bounded denominator property prior to the normalization as it is pointed out by D. Zagier that all forms with algebraic coefficients can be normalized to have integral coefficients.

5.2. For any $n \geq 1$ and with a principal branch fixed, we formally write

$$
(1+x)^{1 / n}=\sqrt[n]{1+x}=\sum_{m \geq 0} \frac{\left(\frac{1}{n}\right)_{m}}{m !} x^{m}
$$

where $\left(\frac{1}{n}\right)_{m}=\frac{1}{n}\left(\frac{1}{n}-1\right) \cdots\left(\frac{1}{n}-m+1\right)$. The next lemma provides a simple but useful criterion for detecting the unbounded denominator property in simple cases.

Lemma 2. Let $n$ be any natural number and $f=1+\sum_{m \geq 1} a(m) w^{m}, a(m) \in$ $\mathbb{Z}$ for all $m$. In terms of (10), we expand $\sqrt[n]{f}=\sum_{m \geq 0} b(m) w^{m}, b(m) \in$ $\mathbb{Z}[1 / n]$. Let $p$ be a prime factor of $n$. If there exists one $b(m)$ which is not p-integral, then

$$
\limsup _{m \rightarrow \infty}-\operatorname{ord}_{p}(b(m)) \rightarrow \infty .
$$

In other words, $\{b(m)\}$ has unbounded denominators.

Proof. It is a special case of [KL06, Lemma 2.7].

\section{3 .}

Example 5. In CdlORV00, Candelas, de la Ossa, and Rodriguez-Villegas have studied the family of quintic Calabi-Yau 3-fold in $\mathbb{P}^{4}$ given by

$$
\sum_{i=1}^{5} x_{i}^{5}-5 \psi x_{1} x_{2} x_{3} x_{4} x_{5}=0
$$

They have shown that

$$
\left.\frac{1}{(5 \psi)^{5}}=w+154 w^{2}+179139 w^{3}+\cdots \in \mathbb{Z}[w]\right], w=e^{2 \pi i t}
$$


where

$$
t=\frac{1}{2 \pi i} \frac{\bar{\omega}_{1}(\psi)}{\bar{\omega}_{0}(\psi)}
$$

is the a ratio of two particular solutions of the Picard-Fuchs equations arising from the quintic family. By the above lemma, the Fourier coefficients of $\psi$ have unbounded denominators.

\section{4 .}

Example 6. Let $\Gamma_{n}$ be as in Example 2. A Hauptmodul of $\Gamma_{n}$ is $t_{n}=\sqrt[n]{t}$ where the expansion of $t$ is as in (8). By Lemma 2, when $n \neq 1,5$, the coefficients of $t_{n}$ have unbounded denominators. Therefore $\Gamma_{n}$ is noncongruence.

Lemma 3. Let $f_{n, k}$ be the weight $k$ cuspform for $\Gamma^{1}(5)$ as in Lemma 1 , then the Fourier coefficients of $1 / f_{n, k}$ have bounded denominators.

Proof. By our choice of $f_{n, k}$ whose poles are supported at the cusps, we know not only $f_{n, k}$ satisfies the bounded denominator property. Take any integral weight normalized newform $g$ for $\Gamma^{1}(5)$. Clearly for any positive integer $m$, both $g^{m}$ and $g^{-m}$ satisfy the bounded denominator property. When $m$ is large enough, $g^{m}\left(f_{n, k}\right)^{-1}$ is also a holomorphic cuspform $h$ for $\Gamma^{1}(5)$, hence $\left(f_{n, k}\right)^{-1}=h g^{-m}$ also satisfies the bounded denominator property.

Proposition 1. Let $f$ be a weight $k>3$ cuspform for some $\Gamma_{n}$ with coefficients in some algebraic number field. If it is not a form for $\Gamma_{1}$ or $\Gamma_{5}$, then the coefficients of $f$ at $\infty$ have unbounded denominators.

Proof. By Lemma 1, $f=f_{n, k} \cdot\left(\sum_{j=1}^{n} a_{j} t_{n}^{j}\right)$ for some $a_{j} \in \mathbb{C}$. Let $F[x] \in \mathbb{C}[x]$ be the nonconstant polynomial with minimal degree such that the coefficients of $F\left(t_{n}\right)$ have bounded denominators. This is $F[x]=x^{n}$ if $5 \nmid n$ or $F[x]=x^{n / 5}$ otherwise. In the first case, if $f \notin S_{k}\left(\Gamma^{1}(5)\right)$, then $a_{j} \neq 0$ for some $j$ not divisible by $n$, hence $\left(\sum a_{j} x^{j}\right)$ is not a polynomial in $x^{n}$, i.e. the Fourier coefficients of $\left(\sum a_{j} t_{n}^{j}\right)$ have unbounded denominators. If $f$ satisfies the bounded denominator property so does $f\left(f_{n, k}\right)^{-1}=\left(\sum a_{j} t_{n}^{j}\right)$ which contradicts our assumption above. The second case can be handled in a similar manner.

5.5. The Fermat curves and modular curves. In the next example, we give one of the realizations of the Fermat curves in terms of modular curves. It is motivated by a question raised by Koblitz (through private conversation) regarding the relations between the Fermat curves and noncongruence subgroups.

For any positive integer $n \geq 2$,

$$
x^{n}+y^{n}=1
$$

is the Fermat curve and its properties have been studied extensively (cf. GR78, KR78, Yui80, Yan96, etc]). Now let $G_{1}$ and $G_{2}$ be two genus 0 
subgroups with Hauptmoduls

$$
x=\sqrt[n]{\lambda}, \quad y=\sqrt[n]{1-\lambda}
$$

respectively, where the lambda function

$$
\begin{aligned}
\lambda(q) & =16 q \prod_{n \geq 1}\left[\left(1+q^{2 n}\right)^{2}\left(1-q^{2 n-1}\right)\right]^{8} \\
& =16 q\left(1-8 q+44 q^{2}-192 q^{3}+718 q^{4}-2400 q^{5}+7352 q^{6}+\cdots\right)
\end{aligned}
$$

is a Hauptmodul for $\Gamma(2)$. (Not just any arbitrary algebraic function of $\lambda$ is another modular function. These two functions can be Hauptmoduls due to the special locations of the zeros and poles of $\lambda$ and $1-\lambda$ ). Clearly $x$ and $y$ satisfy $x^{n}+y^{n}=1$. It is not difficult to see that the Fermat curve above is bi-holomorphic to the modular curve for $\Phi(n)=G_{1} \cap G_{2}$. In the literature, $\Phi(n)$ is the so-called Fermat group. By Lemma 2, it is easy to check that when $n \neq 1,2,4,8$ the Fourier coefficients of the expansions of $x$ and $y$ in $q$ at infinity will have unbounded denominators, which implies $\Phi(n)$ is noncongruence. By using the idea used in the proof of Proposition 1, we can show that when $n \neq 1,2,4,8$, the Fermat group $\Phi(n)$ satisfies the condition (UBD) (cf. Section 5.11).

5.6. Recently, Kurth has verified that for each of the following K3 configurations Kur06

$[1,1,1,1,2,18],[1,1,1,1,10,10],[1,1,2,2,6,12],[1,1,2,2,9,9],[1,1,2,5,5,10]$

$[1,1,4,6,6,6],[2,2,2,3,3,12],[2,2,5,5,5,5],[2,3,3,4,6,6],[3,3,3,3,6,6]$

arising as double covers of rational surfaces mentioned in subsection 2.4 and [TY03, the Fourier coefficients of every possible Hauptmodul of these monodromy groups have unbounded denominators.

5.7. In general, it is relatively easy to construct modular forms for type I character groups whose corresponding homomorphisms send all elliptic and hyperbolic generators to 1 (cf. Example 2 and Lemma 1). To handle all type II or II(A) character groups of a fixed congruence group is more challenging. The following theorem gives a partial positive answer to the open question mentioned at the beginning of this section for type II(A) character groups.

Theorem 6 ([KL06]). Let $\Gamma^{0}$ be any genus 1 congruence subgroup whose modular curve is defined over a number field. If there exists a prime $p$ such that every index-p type II(A) character group of $\Gamma^{0}$ satisfies (UBD), then there exists $c\left(\Gamma^{0}\right)>0$ such that for any $X \gg 0$,

$$
\#\left\{\text { subgroup } \Gamma \text { of } \Gamma^{0} \mid\left[\Gamma^{0}: \Gamma\right]<X \text {, and } \Gamma \text { satisfies }(U B D)\right\}>c\left(\Gamma^{0}\right) \cdot X^{2} \text {. }
$$

It is computationally feasible to check the conditions above. For example $\Gamma^{0}=\Gamma^{0}(11)$ is considered in [KL06] via some results of Atkin in [Atk67]. 


\section{Modularity}

6.1. $l$-adic Scholl representations. Let $\Gamma$ be a noncongruence subgroup with $X_{\Gamma}$ defined over $\mathbb{Q}$ and $k>2$ be an integer. Let $d=\operatorname{dim} S_{k}(\Gamma)$. For every prime $l$, Scholl has constructed a $2 d$-dimensional $\mathbb{Q}_{l}$-vector space $W$ from weight $k$ cuspforms for $\Gamma$ such that there is a continuous homomorphism

$$
\rho: \operatorname{Gal}(\overline{\mathbb{Q}} / \mathbb{Q}) \rightarrow \operatorname{Aut}(W),
$$

(cf. Sch85]). These representations satisfy good properties: They ramify only at a finite set of primes. For each prime $p$ such that $\rho$ is unramified, the characteristic polynomial of the Frobenius element at $p$ has integral coefficients. It has been verified that Scholl's results also hold for odd weight cases (cf. [ $\left.\mathrm{F}^{+} 05\right]$ ). In [Sch88, Scholl investigated the 2-dimensional $l$-adic representation attached to the unique weight 4 genuine cuspform for an index 9 noncongruence subgroup. He showed that it is isomorphic to the $l$-adic representation attached to a weight 4 level 14 congruence newform. Scholl has given a few more unpublished examples of this kind [Sch93].

6.2. In [SB85], Stienstra and Beukers investigated several K3 surfaces with Picard number 20 over $\mathbb{C}$. These K3 surfaces are constructed as double covers of $\mathbb{C} P^{2}$ with branching points along degree 6 polynomials. According to a result of Shioda and Inose [SI77], the L-function of each algebraic K3 surface with Picard number 20 mainly consists of the L-function of a weight 3 congruence Hecke eigenform. In other words, the $l$-adic representations attached the nontrivial part of the $l$-adic second étale cohomology of such a K3 surface are modular. Stienstra and Beukers have identified the Hecke newforms corresponding to each K3 surface in their consideration and have constructed elliptic fibers for those K3 surfaces including the one with configuration [1,1,1,1,10,10]. However, using Sebbar's classification [Seb01, one sees several monodromy groups attached to these elliptic fibrations are noncongruence. Combining the above discussions with the theory of elliptic modular surfaces by Shioda Shi72], we have

Theorem 7. Let $\Gamma$ be a finite index subgroup of the modular group which is conjugate to a monodromy group of a K3 surface over $\mathbb{Q}$ with Picard number 20. Then the l-adic Scholl representation attached to $S_{3}(\Gamma)$ is isomorphic to the l-adic representation attached to certain Hecke eigenform.

This modularity result has significant influence on [LLY05b] and it explains why weight 3 cuspforms are given more attention. As a special case of the above theorem, we know the $l$-adic Scholl representation attached to $S_{3}\left(\Gamma_{2}\right)$ is modular.

6.3. Starting from [LLY05b], investigations have been focused on higher dimensional $l$-adic Scholl representations. In particular, $l$-adic Scholl representations attached to $S_{3}\left(\Gamma_{n}\right)$ have been examined extensively. To study 
these representations, it is natural to decompose the representation spaces into subrepresentation spaces by using available operators. Normalizers of $\Gamma$ in $S L_{2}(\mathbb{R})$ become important as they give rise to involutions on the corresponding $l$-adic representation spaces. Use $\Gamma^{N}$ to denote the group of normalizers of $\Gamma$ in $S L_{2}(\mathbb{R})$. Clearly $\Gamma^{0} \subset \Gamma^{N}$. (M.L. Lang gave an algorithm for finding $\Gamma^{N}$ for a given $\left.\Gamma[\operatorname{Lan} 02]\right)$. Moreover, $\Gamma^{N} / \Gamma$ acts on the finite dimensional spaces $S_{k}(\Gamma)$. We assume the action of any element in $\Gamma^{N} / \Gamma$ is defined over a number field $F$. Consequently, the $l$-adic School representation above, when restricted to the subgroup $\operatorname{Gal}(\overline{\mathbb{Q}} / F)$, can be decomposed into a direct sum of subrepresentations. In some ideal cases, these subrepresentations are 2 -dimensional and are shown to be isomorphic to some $l$-adic Deligne representations attached to congruence newforms [LLY05b, $\mathrm{F}^{+} 05$, ALL05, Lon06, etc].

6.4. With the new exciting progress on major conjectures like the FontaineMazur conjecture [Kis06], and the Serre conjecture [KW06, Kis07], it is promising that one can establish the modularity for a large class of $l$-adic Scholl representations.

\section{Atkin And Swinnerton-Dyer Congruences}

7.1. When $\Gamma$ is a congruence subgroup, we have the following classical result due to Hecke and Petersson. For any positive integer $k$, the space $S_{k}(\Gamma)$ has a basis consisting of Hecke eigenforms. Namely, for each of the basis element $\sum_{n \geq 1} a(n) w^{n}$ there exists a suitable Dirichlet character $\chi$ such that for any prime $p$

$$
a(n p)-a(p) a(n)+\chi(p) p^{k-1} a(n / p)=0, \quad \forall n \in \mathbb{N} .
$$

However, when $\Gamma$ is a noncongruence subgroup, the Hecke operator theory is unsatisfactory as mentioned in Section 4.3. Consequently, such a result no longer holds for noncongruence cuspforms. While working with explicit examples, Atkin and Swinnerton-Dyer have found that three-term recursions like (12) still hold in a $p$-adic sense. What Atkin and Swinnerton-Dyer have observed is the following: given $S_{k}(\Gamma)$, then for almost all primes $p$, the space $S_{k}(\Gamma)$ has a basis (pending on $p$ ) consisting of forms of the form $\sum a(n) w^{n}$ satisfying the ASD congruence relations

$$
a(n p)-A(p) a(n)+B(p) a(n / p)=0 \bmod p^{(k-1)\left(1+\operatorname{ord}_{p} n\right)}, \quad \forall n \geq 1
$$

where $A(p)$ and $B(p)$ are $p$-adic and in general such bases are different for each $p$.

7.2. To examine the ASD congruence relations computationally, Atkin uses " $p$-adic" Hecke operators as. For any "good" prime $p$, define the $p$-adic Hecke operator $T_{p}=T^{2}+s(p) \cdot p^{k-1}$ for $f=\sum a(n) w^{n}$ as follows:

$$
T_{p}(f)=\sum_{n}\left(a(n p)+s(p) p^{k-1} a(n / p)\right) w^{n} .
$$


Here, $s(p)$ is an algebraic number with absolute value 1. In general, it is totally nontrivial to guess the right $s(p)$ values since they are rarely Dirichlet characters. It is called a " $p$-adic" Hecke operator as the $n$th coefficient of $f \mid T_{p}$ will be determined up to modulo some suitable power of $p$ depending on $n$. Unlike the classical Hecke operators which can be diagonalized simultaneously, these operators are a priori defined over various $p$-adic fields. It is clearly exceptional that the space $S_{k}(\Gamma)$ can be decomposed simultaneously for almost all " $p$-adic" Hecke operators.

7.3. In [Sch85], Scholl has proven a collective version of Atkin and SwinnertonDyer's observation. Basically, he showed that for any form $f$ with rational coefficients in a $d$-dimensional space of even weight noncongruence cuspforms, the coefficients of $f$ satisfy a $2 d+1$ term congruence recursion. In [ $\left.\mathrm{F}^{+} 05\right]$, the authors have verified the necessary conditions under which Scholl's results are valid for odd weight cases.

7.4. The available operators remain the key players when one attempts to reduce Scholl's result to 3-term ASD congruence. In [LLY05b, ALL05, Lon06] significant efforts have been made to study the relations between these operators and elements in the Galois groups. With the aid of these operators, stronger results such as the existence of simultaneous (resp. semisimultaneous) eigen basis for Atkin and Swinnerton-Dyer congruences at almost all primes $p$ can be achieved cf. [LLY05b] (resp. [ALL05, Lon06]).

Example 7 ([LLY05b]). There exists a basis $f_{ \pm}=\sum a_{ \pm}(n) w^{n}$ with coefficients in $\mathbb{Z}[1 / 3](i)$ for $S_{3}\left(\Gamma_{3}\right)$ and a pair of newforms $g_{ \pm}=\sum b_{ \pm}(n) q^{n}$ with coefficients in $\mathbb{Z}[i]$ such that for any $n \geq 1$ and any odd prime $p$ different from 3

$$
\frac{a_{ \pm}(n p)-b_{ \pm}(p) a_{ \pm}(n)+\left(\frac{-3}{p}\right) p^{2} a_{ \pm}(n / p)}{(n p)^{2}}
$$

is integral for some prime in $\mathbb{Z}[i]$ above $p$.

In general, the symmetries inherited from $\Gamma^{N} / \Gamma$ is not sufficient to derive 3term Atkin and Swinnerton-Dyer congruences, especially when the dimension $d$ is large [ALo07]. It is still widely open whether the original 3-term Atkin and Swinnerton-Dyer congruences always hold for any space of noncongruence cuspforms.

\section{REFERENCES}

[AL70] A. O. L. Atkin and J. Lehner, Hecke operators on $\Gamma_{0}(m)$, Math. Ann. 185 (1970), 134-160.

[ALo07] A. O. L. Atkin and L. Long, On Atkin and Swinnerton-Dyer congruence relations for some noncongruence cuspforms.

[ALL05] A. O. L. Atkin, W.C. Li, and L. Long, On Atkin and Swinnerton-Dyer congruence relations (2), math.NT/0512614, preprint (2005), to apper on Math. Ann. 
[ASD71] A. O. L. Atkin and H. P. F. Swinnerton-Dyer, Modular forms on noncongruence subgroups, Combinatorics (Proc. Sympos. Pure Math., Vol. XIX, Univ. California, Los Angeles, Calif., 1968), Amer. Math. Soc., Providence, R.I., 1971, pp. 1-25.

[Atk67] A. O. L. Atkin, Proof of a conjecture of Ramanujan, Glasgow Math. J. 8 (1967), 14-32.

[BCDT] C. Breuil, B. Conrad, F. Diamond, and R. Taylor, On the modularity of elliptic curves over $\mathbf{Q}$ : wild 3-adic exercises, J. Amer. Math. Soc.

[Bea82] A. Beauville, Les familles stables de courbes elliptiques sur $\mathbf{p}^{1}$ admettant quatre fibres singulières, C. R. Acad. Sci. Paris Sér. I Math. 294 (1982), no. 19, $657-660$.

[Bel79] G. V. Belyŭ, Galois extensions of a maximal cyclotomic field, Izv. Akad. Nauk SSSR Ser. Mat. 43 (1979), no. 2, 267-276, 479.

[Ber94] G. Berger, Hecke operators on noncongruence subgroups, C. R. Acad. Sci. Paris Sér. I Math. 319 (1994), no. 9, 915-919.

[Ber00] , Relations between cusp forms on congruence and noncongruence groups, Proc. Amer. Math. Soc. 128 (2000), no. 10, 2869-2874.

[Bir94] B. Birch, Noncongruence subgroups, covers and drawings, The Grothendieck theory of dessins d'enfants (Luminy, 1993), London Math. Soc. Lecture Note Ser., vol. 200, Cambridge Univ. Press, Cambridge, 1994, pp. 25-46.

[BLS64] H. Bass, M. Lazard, and J.-P. Serre, Sous-groupes d'indice fini dans $\mathbf{S L}(n, \mathbf{Z})$, Bull. Amer. Math. Soc. 70 (1964), 385-392.

[BPVdV84] W. Barth, C. Peters, and A. Van de Ven, Compact complex surfaces, Ergebnisse der Mathematik und ihrer Grenzgebiete (3) [Results in Mathematics and Related Areas (3)], vol. 4, Springer-Verlag, Berlin, 1984.

[Car71] P. Cartier, Groupes formels, fonctions automorphes et fonctions zeta des courbes elliptiques, Actes du Congrès International des Mathématiciens (Nice, 1970), Tome 2, Gauthier-Villars, Paris, 1971, pp. 291-299.

[CdlORV00] P. Candelas, X. de la Ossa, and F. Rodriguez-Villegas, Calabi-Yau manifolds over finite fields. I, hep-th/0012233, 2000.

[CLY04] K. S. Chua, M. L. Lang, and Y. Yang, On Rademacher's conjecture: congruence subgroups of genus zero of the modular group, J. Algebra 277 (2004), no. $1,408-428$.

[CP03] C. J. Cummins and S. Pauli, Congruence subgroups of $\operatorname{PSL}(2, \mathbb{Z})$ of genus less than or equal to 24, Experiment. Math. http://www.math.tu-berlin.de/ p pauli/congruence/ 12 (2003), no. 2, $243-255$.

[Den75] J. B. Dennin, Jr., The genus of subfields of $K(n)$, Proc. Amer. Math. Soc. 51 (1975), 282-288.

[Dit76] B. Ditters, Sur les congruences d'Atkin et de Swinnerton-Dyer, C. R. Acad. Sci. Paris Sér. A-B 282 (1976), no. 19, Ai, A1131-A1134.

[DS05] F. Diamond and J. Shurman, A first course in modular forms, Graduate Texts in Mathematics, vol. 228, Springer-Verlag, New York, 2005.

$\left[\mathrm{F}^{+} 05\right] \quad$ L. Fang, J. W. Hoffman, B. Linowitz, A. Rupinski, and Verrill H., Modular forms on noncongruence subgroups and Atkin-Swinnerton-Dyer relations, preprint (2005).

[Fri86] R. Fricke, Über die Substitutionsgruppen, welche zu den aus dem Legendre'schen Integralmodul k2(w) gezogenen Wurzeln gehören. (Mit einer Figurentafel), Math. Ann. 28 (1886), 99-118.

[Gel84] S. Gelbart, An elementary introduction to the Langlands program, Bull. Amer. Math. Soc. (N.S.) 10 (1984), no. 2, 177-219. 
[GR78] B. H. Gross and D. E. Rohrlich, Some results on the Mordell-Weil group of the Jacobian of the Fermat curve, Invent. Math. 44 (1978), no. 3, 201-224.

[Hec37] E. Hecke, Über Modulfunktionen und die Dirichletschen Reihen mit Eulerscher Produktentwicklung. I and II, Math. Ann. 114 (1937), no. 1, 1-28,316-351.

[HL05] P. Hubert and S. Lelièvre, Noncongruence subgroups in $\mathcal{H}(2)$, Int. Math. Res. Not. (2005), no. 1, 47-64.

[Hon70] T. Honda, On the theory of commutative formal groups, J. Math. Soc. Japan 22 (1970), 213-246.

[Hsu96] T. Hsu, Identifying congruence subgroups of the modular group, Proc. Amer. Math. Soc. 124 (1996), no. 5, 1351-1359.

[Jon79] G. A. Jones, Triangular maps and noncongruence subgroups of the modular group, Bull. London Math. Soc. 11 (1979), no. 2, 117-123.

[Jon86] _ Congruence and noncongruence subgroups of the modular group: a survey, Proceedings of groups - St. Andrews 1985 (Cambridge), London Math. Soc. Lecture Note Ser., vol. 121, Cambridge Univ. Press, 1986, pp. 223234.

[JS94] G. Jones and D. Singerman, Maps, hypermaps and triangle groups, The Grothendieck theory of dessins d'enfants (Luminy, 1993), London Math. Soc. Lecture Note Ser., vol. 200, Cambridge Univ. Press, Cambridge, 1994, pp. $115-145$.

[KW06] C. Kare, J-P. Wintenburger, Serre's modularity conjecture, The case of odd conductor I and II, preprint (2006)

[Kat81] N. M. Katz, Crystalline cohomology, Dieudonné modules, and Jacobi sums, Automorphic forms, representation theory and arithmetic (Bombay, 1979), Tata Inst. Fund. Res. Studies in Math., vol. 10, Tata Inst. Fundamental Res., Bombay, 1981, pp. 165-246.

[Kis06] M. Kisin, The fontaine-mazur conjecture for gl2, preprint, http://www.math.uchicago.edu/ kisin/preprints.html (2006).

[Kis07] M. Kisin, Modularity of 2-adic Barsotti-Tate representations, http://www.math.uchicago.edu/ kisin/preprints.html (2007).

[KN65] M. I. Knopp and M. Newman, Congruence subgroups of positive genus of the modular group, Illinois J. Math. 9 (1965), 577-583.

[Kob93] N. Koblitz, Introduction to elliptic curves and modular forms, second ed., Springer-Verlag, New York, 1993.

[Kod63] K. Kodaira, On compact analytic surfaces. II, III, Ann. of Math. (2) 77 (1963), 563-626; ibid. 78 (1963), 1-40.

[KR78] N. Koblitz and D. Rohrlich, Simple factors in the Jacobian of a Fermat curve, Canad. J. Math. 30 (1978), no. 6, 1183-1205.

[Kul85] R. S. Kulkarni, A new proof and an extension of a theorem of Millington on the modular group, Bull. London Math. Soc. 17 (1985), no. 5, 458-462.

[Kur06] C. Kurth, URL: http://orion.math.iastate.edu/linglong/Noncong/noncong.htm (2006).

[KL06] C. A. Kurth and L. Long, On modular forms for some noncongruence arithmetic subgroups, math.NT/0612100 (2006).

[Lan02] M. L. Lang, Normalisers of subgroups of the modular group, J. Algebra 248 (2002), no. 1, 202-218.

[Li75] W.C. Li, Newforms and functional equations, Math. Ann. 212 (1975), 285315.

[Li96] _ Number theory with applications, World Scientific Publishing Co. Inc., River Edge, NJ, 1996. 
[LLY05a] W.C Li, L. Long, and Z. Yang, Modular forms for noncongruence subgroups, Quarterly Journal of Pure and Applied Mathematics 1 (2005), no. 1, 205-221.

[LLY05b] On Atkin and Swinnerton-Dyer congruence relations, J. of Number Theory 113 (2005), no. 1, 117-148.

[Lon06] L. Long, On Atkin and Swinnerton-Dyer congruence relations (3), math.NT/0701310 (2006).

[MB] F. Montanus and F. Beukers, http://www.math.uu.nl/people/beukers/mirandapersson.

[Mil69a] M. H. Millington, On cycloidal subgroups of the modular group, Proc. London Math. Soc. (3) 19 (1969), 164-176.

[Mil69b] , Subgroups of the classical modular group, J. London Math. Soc. (2) 1 (1969), 351-357.

[Miy71] T. Miyake, On automorphic forms on $\mathrm{GL}_{2}$ and Hecke operators, Ann. of Math. (2) 94 (1971), 174-189.

[Mor17] L. J. Mordell, On Mr. Ramanujan's empirical expansions of modular functions, Proc. Cambridge Phil. Soc. 19 (1917), 117-124.

[MP89] R. Miranda and U. Persson, Configurations of $I_{n}$ fibers on elliptic K3 surfaces, Math. Z. 201 (1989), no. 3, 339-361.

[Mur93] M. R. Murty, A motivated introduction to the Langlands program, Advances in number theory (Kingston, ON, 1991), Oxford Sci. Publ., Oxford Univ. Press, New York, 1993, pp. 37-66.

[Nér64] A. Néron, Modèles minimaux des variétés abéliennes sur les corps locaux et globaux, Inst. Hautes Études Sci. Publ.Math. No. 21 (1964), 128.

[New65] M. Newman, Normal subgroups of the modular group which are not congruence subgroups, Proc. Amer. Math. Soc. 16 (1965), 831-832.

[Nor85] M. Nori, On certain elliptic surfaces with maximal Picard number, Topology 24 (1985), no. 2, 175-186.

[Ogg69] A. P. Ogg, Modular forms and Dirichlet series, W. A. Benjamin, Inc., New York-Amsterdam, 1969.

[Pet39] H. Petersson, Konstruktion der sämtlichen Lösungen einer Riemannschen Funktionalgleichung durch Dirichletreihen mit Eulerscher Produktentwicklung I, Math. Ann. 116 (1939), no. 1, 401-412.

[Pic86] G. Pick, Über gewisse ganzzahlige lineare Substitutionen, welche sich nicht durch algebraische Congruenzen erklären lassen, Math. Ann. 28 (1886), 119124 .

[Ran67] R. A. Rankin, Lattice subgroups of free congruence groups, Invent. Math. 2 (1967), 215-221.

[Ran77] Modular forms and functions, Cambridge University Press, Cambridge, 1977.

[Rei58] I. Reiner, Normal subgroups of the unimodular group, Illinois J. Math. 2 (1958), 142-144.

[Sar90] P. Sarnak, Some applications of modular forms, Cambridge Tracts in Mathematics, vol. 99, Cambridge University Press, Cambridge, 1990.

[SB85] J. Stienstra and F. Beukers, On the Picard-Fuchs equation and the formal Brauer group of certain elliptic K3-surfaces, Math. Ann. 271 (1985), no. 2, 269-304.

[Sch85] A. J. Scholl, Modular forms and de Rham cohomology; Atkin-SwinnertonDyer congruences, Invent. Math. 79 (1985), no. 1, 49-77.

[Sch88]_ The l-adic representations attached to a certain noncongruence subgroup, J. Reine Angew. Math. 392 (1988), 1-15. 
, The l-adic representations attached to non-congruence subgroups $i i$, preprint (1993).

[Sch97] _ On the Hecke algebra of a noncongruence subgroup, Bull. London Math. Soc. 29 (1997), no. 4, 395-399.

[Sch04] - On some l-adic representations of galois group attached to noncongruence subgroups, math.NT/0402111 (2004).

[Seb01] A. Sebbar, Classification of torsion-free genus zero congruence groups, Proc. Amer. Math. Soc. 129 (2001), no. 9, 2517-2527 (electronic).

[Seb02] - Modular subgroups, forms, curves and surfaces, Canad. Math. Bull. 45 (2002), no. 2, 294-308.

[Sel65] A. Selberg, On the estimation of Fourier coefficients of modular forms, Proc. Sympos. Pure Math., Vol. VIII, Amer. Math. Soc., Providence, R.I., 1965, pp. 1-15.

[Shi71] G. Shimura, Introduction to the arithmetic theory of automorphic functions, Publications of the Mathematical Society of Japan, No. 11. Iwanami Shoten, Publishers, Tokyo, 1971, Kanô Memorial Lectures, No. 1.

[Shi72] T. Shioda, On elliptic modular surfaces, J. Math. Soc. Japan 24 (1972), 20 59.

[SI77] T. Shioda and H. Inose, On singular K3 surfaces, Complex analysis and algebraic geometry, Iwanami Shoten, Tokyo, 1977, pp. 119-136.

[Sti85] J. Stienstra, Les groupes formels d'Artin-Mazur et les congruences d'AtkinSwinnerton-Dyer, Study group on ultrametric analysis, 12th year, 1984/85, No. 2, Secrétariat Math., Paris, 1985, pp. Exp. No. 18, 13.

[Sti05] - Mahler measure variations, eisenstein series and instanton expansions, to appear in the Proceedings of the 2003 Banff workshop on Calabi-Yau Varieties and Mirror Symmetry, math.NT/0502193 (2005).

[Sto84] W. W. Stothers, Level and index in the modular group, Proc. Roy. Soc. Edinburgh Sect. A 99 (1984), no. 1-2, 115-126.

[Tho80] J. G. Thompson, A finiteness theorem for subgroups of $\operatorname{PSL}(2, \mathbf{R})$ which are commensurable with $\operatorname{PSL}(2, \mathbf{Z})$, The Santa Cruz Conference on Finite Groups (Univ. California, Santa Cruz, Calif., 1979), Proc. Sympos. Pure Math., vol. 37, Amer. Math. Soc., Providence, R.I., 1980, pp. 533-555.

[Tho89]__ Hecke operators and noncongruence subgroups, Group theory (Singapore, 1987), de Gruyter, Berlin, 1989, Including a letter from J.-P. Serre, pp. $215-224$.

[TW95] R. Taylor and A. Wiles, Ring-theoretic properties of certain Hecke algebras, Ann. of Math. (2) 141 (1995), no. 3, 553-572.

[TY03] J. Topp and N. Yui, Explicit equations of some elliptic modular surfaces, math.AG/0307230 (2003).

[Wil95] A. Wiles, Modular elliptic curves and Fermat's last theorem, Ann. of Math. (2) 141 (1995), no. 3, 443-551.

[Woh64] K. Wohlfahrt, An extension of F. Klein's level concept, Illinois J. Math. 8 (1964), 529-535.

[Yan96] T. Yang, Cusp forms of weight 1 associated to Fermat curves, Duke Math. J. 83 (1996), no. 1, 141-156.

[Yui80] N. Yui, On the Jacobian variety of the Fermat curve, J. Algebra 65 (1980), no. $1,1-35$.

[Zog91] P. Zograf, A spectral proof of Rademacher's conjecture for congruence subgroups of the modular group, J. Reine Angew. Math. 414 (1991), 113-116. 
Department of Mathematics, Iowa State University, Ames, IA 50011, USA

E-mail address: linglong@iastate.edu 